\title{
Research of Improved Active Frequency Drift with Positive Feedback Islanding Detection Method
}

\author{
LIU Guan-qi, SHAO Long, HU Ting \\ Department of Electrical Engineering \\ North China Electric Power University \\ Baoding, China \\ shaolong211@126.com
}

\begin{abstract}
Nowadays, the active frequency drift with positive feedback (AFDPF) islanding detection method which can solve the islanding of photovoltaic (PV) systems is widely used. However, the traditional AFDPF method may cause some problems such as the missing detection of islanding and the extension of detection time when the power grid fails and the loads' natures are different. In order to solve the problems, the essay proposes an improved AFDPF islanding detection method. This method introduces sign-function before the disturbance signal $c f_{0}$ (initial chopping fraction). Through this way, we can make correction for $c f_{0}$ so as to overcome the problems mentioned above. To verify the validity of the method, an example is utilized. The simulation analysis of the example indicates that after introducing sign-function before $c f_{0}$, the islanding can be detected effectively under different loads conditions, in addition, the detection time can be reduced a lot and the grid-connected current waveform is quite good. So by this method, both the detection reliability and the detection efficiency can be improved greatly.
\end{abstract}

Keywords-Photovoltaic system grid-connected; Islanding detection; Positive feedback; Active frequency drift;

\section{INTRODUCTION}

As a renewable energy, solar energy has aroused increasing attention of governments and energy experts around the world these years. In the domestic, electricity shortage has been a serious problem, the grid-connected photovoltaic (PV) power generation system is expected to alleviate the tense situation in the future. When an increasing number of PV power generation systems are connected to the power grid, it brings a new phenomenon in grid. That is protection-islanding effect. Islanding effect refers to customers' grid-connected inverter is still in working condition while the power company stops power supply because of grid failure or power outage maintenance, which makes the grid-connected inverter and the loads around it form a power self-supply network which power company can not control. Islanding effect can cause the electrical equipment damage and personal injury, so the occurrence of islanding effect must be promptly detected and the connection between system and grid must be cut off.

At the beginning, the paper introduces the principle of AFD and AFDPF. Because there are large differences on detection effect of different loads with traditional AFDPF islanding detection method. So next, this paper introduces an improved AFDPF islanding detection method and analyzes the working principles as well as the influence of the loads' natures on the detection method. At last, through simulation, the effectiveness of the method used is verified.

\section{AFD WORK PRINCIPLE}

The active frequency drift (AFD) method is a commonly used active islanding detection method. Through taking a sample of the public node's frequency and offsetting this frequency, it can be current output frequency of the inverter, resulting in disturbance to the load's voltage frequency $^{[1]}$. As shown in Figure 1.

The AFD method sets the frequency of the grid current slightly higher than that of the grid voltage. If the current half-wave has been completed while the voltage is not zero, then the AFD forces the current value to be zero. Until the arrival of the voltage zero point, the current starts the next half-wave. When the power is cut off, the voltage frequency of common node which is affected by the current frequency deviates from the original value, and when the frequency exceeds normal range, this method can detect islanding. This method has a few advantages, such as small impacts on the power quality, easy to implement and so on ${ }^{[2]}$.

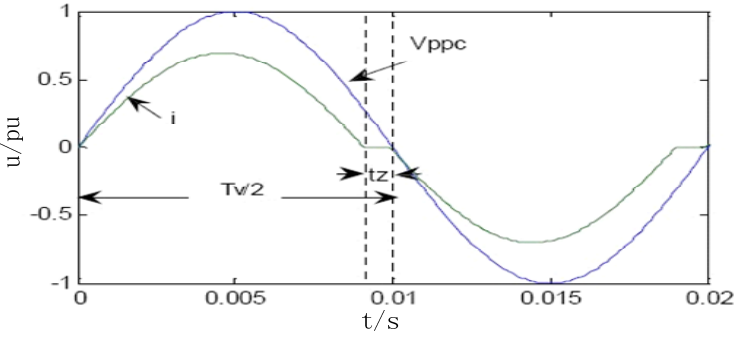

Fig. 1 The diagram of AFD detection method

In Figure 1, $V_{\mathrm{pcc}}$ represents the voltage of common coupling point as and when it comes to the condition of connected-grid operation, it is the grid voltage. $T_{\mathrm{v}}$ represents the corresponding cycle, $i$ represents the current output of the inverter, $t_{\mathrm{z}}$ represents the current chopping time, $c f=2 t_{z} / T_{V}$ represents the chopping fraction. Through Fourier Analysis, we can get that the inverter's current output is $w t_{z} / 2$ ahead than the fundamental current on phase. $w t_{z} / 2$ is defined as active frequency drift angle $\left(\theta_{A F D}\right)^{[1]}$. That is:

$$
\theta_{\text {AFD }}=\omega t_{z} / 2=\frac{1}{2}(2 \pi) \frac{c f}{2}=\frac{\pi}{2} c f
$$

$\theta_{\text {load }}$ is defined as the angle that load's current surpass 
the voltage. That is:

$$
\theta_{\text {load }}=\tan ^{-1}\left[R\left(\omega C-\frac{1}{\omega L}\right)\right]=\tan ^{-1}\left[Q_{f}\left(\frac{f}{f_{0}}-\frac{f_{0}}{f}\right)\right]
$$

If $\theta_{A F D}$ is always greater or less than the $\theta_{\text {load }}$ after off-grid, the frequency will drift in just one direction. The stable operating point does not exist so that the islanding can be detected.

However, as for the parallel $R L C$ loads, no matter the loads' impedance angle is greater or less than zero, the function offsets because of the mutual influence of the impedance angle and frequency drift. And for both the frequency and voltage fail to exceed the preset threshold, the system will be unable to detect the appearance of islanding phenomenon.

\section{TRADITIONAL POSITIVE FEEDBACK AFD DETECTION PRINCIPLE}

AFD detection has following requirements ${ }^{[3]}$ : (1) Having small impacts on power quality under grid-connected operation. (2) Detecting the islanding quickly. (3) Having small detection blind area or no blind area in particular loads. In order to meet the above requirements, AFD method introduces the positive feedback of frequency. That is Active Frequency with Positive Feedback (AFDPF). Its expression is:

$$
c f_{k}=c f_{0}+k\left(f-f_{g}\right)
$$

$c f_{0}$ is initial chopping fraction, $k$ is positive feedback fraction of frequency, $f_{g}$ is grid frequency. Substitute formula (3) into formula (1):

$$
\theta_{\text {AFDPF }}=\frac{\pi}{2} c f_{k}=\frac{\pi}{2}\left[c f_{0}+k\left(f-f_{g}\right)\right]
$$

$c f_{0}$ represents the natural frequency disturbance when grid-connected, and its function is to trigger the frequency drift at the moment of off-grid. The value of $c f_{0}$ affects the output current's harmonic wave directly. In general, $c f_{0}$ and $\mathrm{k}$ are taken to be 0.02 and 0.1 respectively.

\section{IMPROVED POSITIVE FEEDBACK AFD PRINCIPLE}

In traditional AFDPF islanding detection method, the disturbance signal $c f$ disturbs $f$ in just one direction. When the grid fails and the nature of load is different, the change direction of $f$ may be contrary to the disturbance signal's direction, which will slow the errors accumulation of $f$, thus extending the islanding detection time. Under special conditions, the balancing effect of load on the $f$ can offset frequency disturbances, in which case there will be the missing detection of islanding.

Taking the presence of circuit noise and detection errors into consideration, the testing frequency always drifts compared to the grid frequency at the moment of off-grid. Therefore, the initial chopping fraction can be amended: $c f_{k}=c f_{0}+k \Delta f=\operatorname{sign}\left(f-f_{g}\right) \times c f_{0}+k\left(f-f_{g}\right)$

When $f-f_{g} \geq 0, \operatorname{sign}\left(f-f_{g}\right)=1$, when $f-f_{g}<0, \operatorname{sign}\left(f-f_{g}\right)=-1$. So, the islanding detection efficiency for both inductive and capacitive load can be improved.

\section{THE IMPROVED AFDPF DETECTION METHOD FOR SIMULATION}

\section{A. Simulation model}

According to the theoretical analysis above, the connected-grid inverter system's power output level simulation model in $2 \mathrm{~kW}$ single-phase photovoltaic power generation system is established in Matlab/Simulink. The simulation model consists of the main circuit section, the connected-grid control section and islanding detection section in the output level of the single-phase grid system. Figure 2, 3, 4 are the three-part simulation module respectively. In islanding detection module, the function of AFDPF module is realized by the s-function function. Grid current and grid voltage are same in frequency and phase $^{[4][5][6]}$.

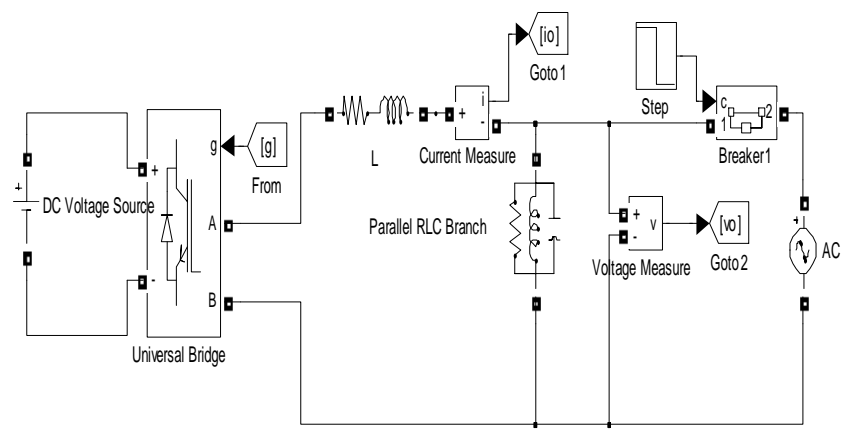

Fig.2 Main circuit block diagram

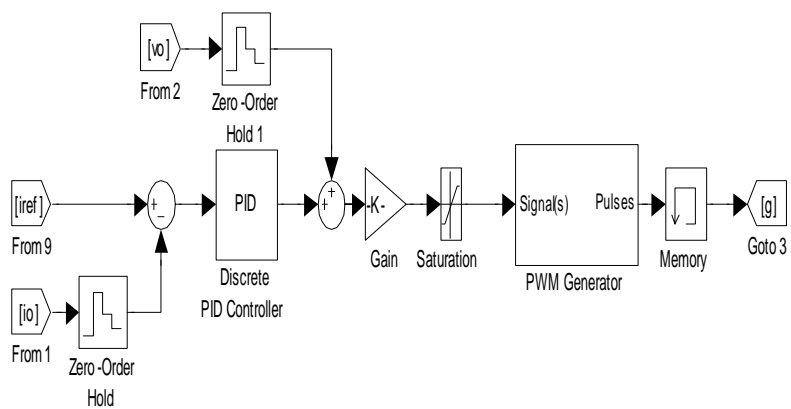

Fig.3 Grid control block diagram

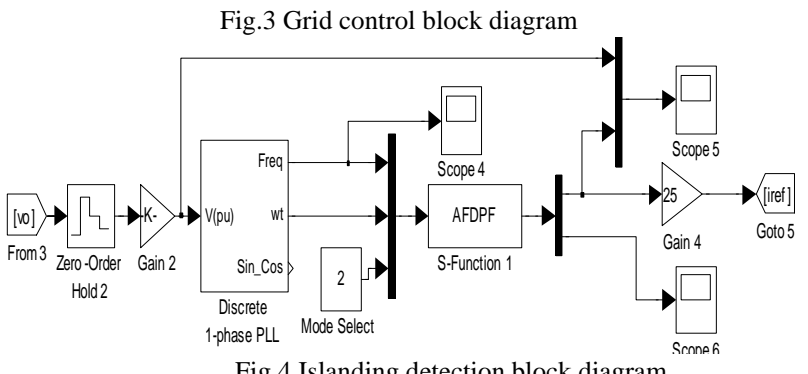

Fig.4 Islanding detection block diagram 


\section{B. Analysis of simulation results}

Take the simulation parameters: DC voltage $400 \mathrm{~V}$, the grid voltage RMS 220V. Frequency is $50.0 \mathrm{~Hz}$. Reference value of the inverter current output is $12.5 \mathrm{~A}$. The action threshold of frequency protection is $50.0 \pm 0.5 \mathrm{~Hz}$. The load's active power is $2 \mathrm{~kW}$. Filter inductor $L=5 \mathrm{mH}, R=0.01 \Omega$. Setting load quality factor $Q_{f}=2.5 \cdot$ RLC load parameters as follows: $R=24.04 \Omega, C=1.325 \times 10^{-6} F, L=7.65 \times 10^{-3} H$, Resonant frequency $f_{0}=50 \mathrm{~Hz}$. Grid breaks off at $0.12 \mathrm{~s}$.

To observe easily, peak value of voltage in figure is $1 / 8$ of the actual value. The current amplitude is expanded to 2 times of the original value. Depending on the different $c f_{0}$ values, there will be three times of simulation respectively.

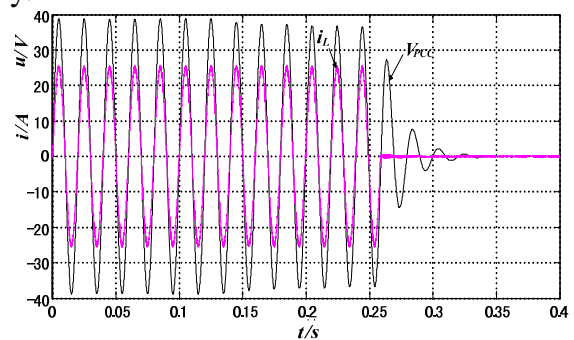

(a) Load voltage and grid current of PCC point

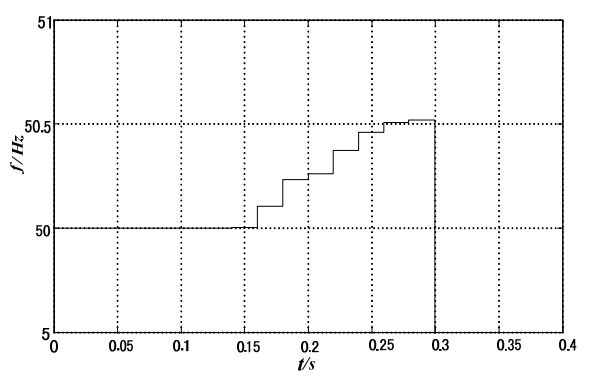

(b) Voltage frequency of PCC point

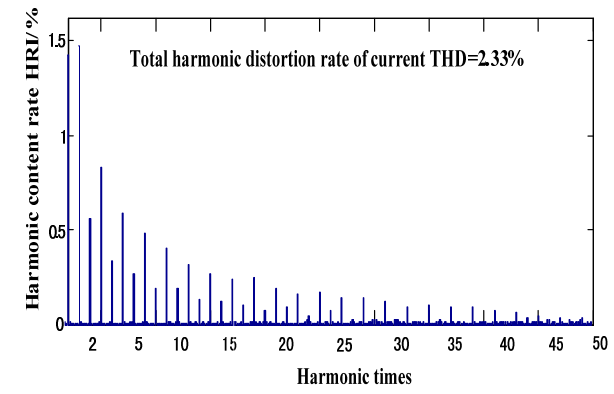

(c) Grid-connected current THD

Fig.5 Islanding detection when $c f=0.02+0.1 \cdot\left(f-f_{g}\right)$

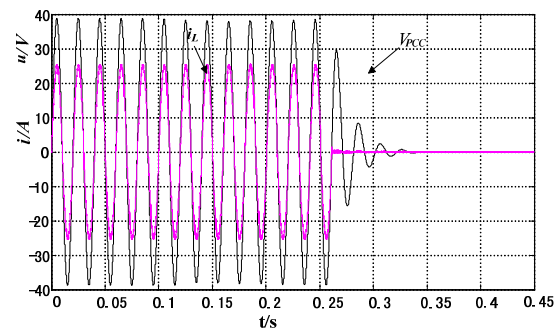

(a) Load voltage and grid current of PCC point

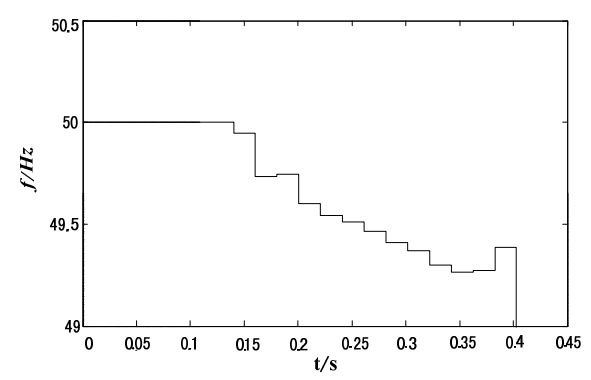

(b)Voltage frequency of PCC point

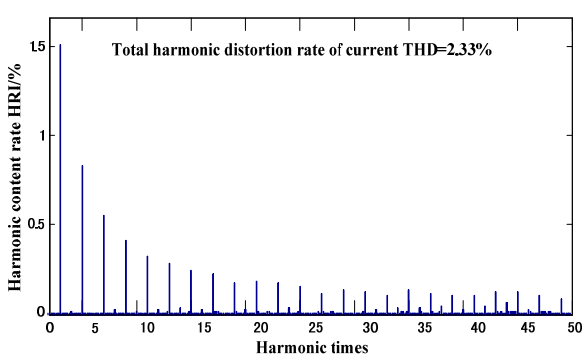

(c) Grid-connected current THD

Fig .6 Islanding detection when $c f=-0.02+0.1 \cdot\left(f-f_{g}\right)$

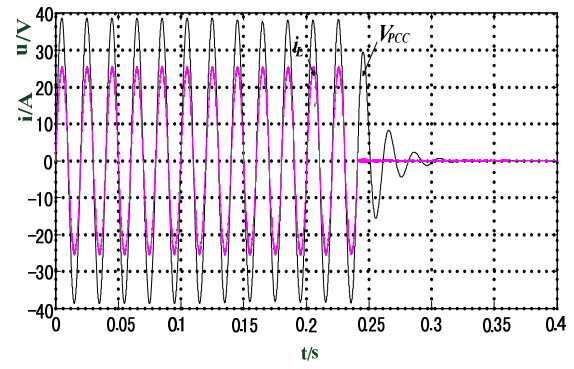

(a) Load voltage and grid current of PCC point

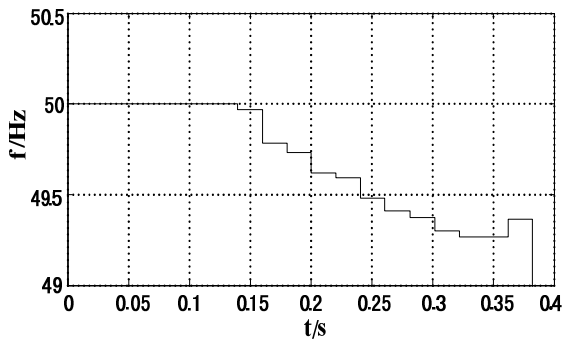

(b)Voltage frequency of PCC point 